\title{
III-Nitride Nanowires Grown on the Graphene
}

\author{
Xiangqian Xiu, Yuewen Li, Lin Chen, Xuemei Hua, Zili Xie, Peng Chen, Rong Zhang, Youdou Zheng \\ School of Electronic Science \& Engineering, Nanjing University \\ Hankou road 22\#, Nanjing 210093, China \\ xqxiu@nju.edu.cn
}

\section{Extended Abstract}

Group III-nitride semiconductors such as GaN, InN, and AlN are widely applied in optoelectronics, high frequency and high-power electronics, such as FETs, LEDs, solar cells, terahertz emitters and detectors etc. Recently, group IIInitride nanowires (NWs) have been an advanced research hotspot because the effects induced by the lattice and thermal mismatches, such as dislocations and stress etc., can be largely eliminated in nanowires. Graphene has been widely studied because of its high thermal and mechanical stability, flexibility, high carrier mobility etc. And it also has been an ideal intermediate layer to grow nitride nanowires to reduce stress because of its sixfold symmetry [1].

In this paper, we present the growth of nitride NWs on the graphene by a simple metal-sublimation CVD method with low-cost and higher growth rate. Metal (gallium or indium) and ammonia are as the reactants. The effect of the catalyst ( $\mathrm{Au}$ or $\mathrm{Ni}$ ) and graphene on the growth of nitride NWs is investigated, including the growth process with or without catalyst or graphene. GaN/InN nanowires were characterized by scanning electron microscopy (SEM), photoluminescence (PL), X-ray diffraction (XRD), Raman spectra and transmission electron microscopy (TEM).

High quality GaN NWs were grown on graphene/sapphire substrates by CVD method. The effects of temperature, buffer layer and catalyst on GaN NWs were studied in details. The results showed that GaN NWs with uniform diameter could be obtained without catalyst in the proper $\mathrm{NH}_{3}$ flow rate. The smooth GaN NWs are densely distributed on the graphene/GaN/sapphire substrate when the reaction time is $5 \mathrm{~min}$. Raman spectra $\mathrm{E}_{2}$ (high) peak of GaN NWs is similar to that of the bulk GaN, which mean that the stress between GaN and sapphire has been eliminated because of the introduction of graphene. GaN buffer layer was grown on the surface of graphene/GaN/sapphire substrates at a low temperature and then heated up to $1100^{\circ} \mathrm{C}$ for the growth of GaN NWs, in order to obtain a nanowire structure with preferential orientation. And the results show that graphene and buffer layer play important roles in obtaining vertically ordered arrays of $\mathrm{GaN}$ nanowires.

Stress-free InN NWs were grown on Au/graphene/GaN/sapphire substrate by In-sublimation CVD method. The phonon $\mathrm{E}_{2}$ (high) and $\mathrm{A}_{1}$ (LO) mode in Raman spectra is observed respectively at 490.8 and $585 \mathrm{~cm}^{-1}$ for InN with graphene inter-layer, as expected similar to that of bulk InN. The inter-planar spacing of (0002) and (11-20) are $2.85 \AA$ and $1.77 \AA$ by the high resolution TEM image of InN NWs, according with the XRD result, which are closer to the theoretical value, confirming that the stress originated from hetero-epitaxy was release by graphene inter-layer.

In conclusion, the graphene may play a critical role together with the catalyst for the growth of nitride NWs with preferential orientation. Au/Ni-catalyst assist the growth of nitride NWs, and the stress in NWs can be released by the aid of graphene inter-layer. This study will promote the rapid and low-cost growth of high-quality nitride nanowires for high performance devices.

\section{References}

[1] C. Kunook, B. Hyeonjun, T. Youngbin et al. "Growth and characterizations of GaN micro-rods on graphene films for flexible light emitting diodes," APL Materials, vol. 2, p. 092512, 2014. 